\title{
Oscillation of Nabla Difference Equations with Several Delay Arguments
}

\author{
Hongwu Wu \\ South China University of Technology \\ School of Mathematics \\ Guangzhou 510640, China \\ Julio G. Dix \\ Texas State University \\ Department of Mathematics \\ 601 University Dr. \\ San Marcos, TX 78666, USA
}

\begin{abstract}
This article concerns the oscillation of solutions to first-order linear nabla difference (backwards difference) equations with several delay arguments and oscillatory coefficients. We present oscillation criteria through iterated estimates involving all delay arguments. We give examples that illustrate the interaction among the delay arguments, and present numerical examples using Matlab software. It should be noted that these results parallel those obtained for differential equations by $\mathrm{Wu}$ and Dix in [14].
\end{abstract}

AMS Subject Classifications: 39A10, 39A21, 34C10.

Keywords: Oscillation, nabla difference, backward difference, multiple delay arguments.

\section{Introduction}

Let $\mathbb{N}_{a}=\{a, a+1, a+2, \ldots\}$ and $\mathbb{N}_{a}^{b}=\{a, a+1, a+2, \ldots, b\}$, where $a, b \in \mathbb{R}$ and $b-a$ is a positive integer. For a function $f: \mathbb{N}_{a} \rightarrow \mathbb{R}$, we define the nabla operator by

Received June 3, 2019; Accepted July 15, 2019

Communicated by Martin Bohner 
$\nabla f(t):=f(t)-f(t-1)$ for $t \in \mathbb{N}_{a+1}$. In some publications this operator is called the backward difference operator, while the forward difference operator is denoted by $\Delta f(t):=f(t+1)-f(t)$.

In this article, we are concerned with the oscillation properties of solutions to the first-order linear nabla difference equations with several delay arguments

$$
\nabla x(t)+\sum_{i=1}^{n} p_{i}(t) x\left(\tau_{i}(t)\right)=0, \quad t \in \mathbb{N}_{0},
$$

where $p_{i}: \mathbb{N}_{0} \rightarrow \mathbb{R}, \tau_{i}: \mathbb{N}_{0} \rightarrow \mathbb{Z}$ are non-decreasing and satisfy $\tau_{i}(t) \leq t-1$ and $\lim _{t \rightarrow \infty} \tau_{i}(t)=\infty$ for $i \in \mathbb{N}_{1}^{n}$.

The following notation will be used in this paper. The raising operator is defined as

$$
t^{\bar{r}}:=t(t+1)(t+2) \cdots(t+r-1), \quad r \in \mathbb{N}_{1}, t \in \mathbb{R} .
$$

From this definition and the nabla operator, we obtain the power rule

$$
\nabla_{t}\left(-\frac{1}{r+1}(1-\sigma(t))\right)^{\overline{r+1}}=(1-t)^{\bar{r}}
$$

where $\sigma(t)=t+1$. Assume $f: \mathbb{N}_{a+1} \rightarrow \mathbb{R}$ and $b \in \mathbb{N}_{a}$. Then the nabla integral of $f$ from $a$ to $b$ is defined by

$$
\int_{a}^{b} f(t) \nabla t:=\sum_{t=a+1}^{b} f(t), \quad t \in \mathbb{N}_{a} .
$$

We now state the fundamental theorem for the nabla calculus. Assume $f: \mathbb{N}_{a+1}^{b} \rightarrow \mathbb{R}$ and $F: \mathbb{N}_{a}^{b} \rightarrow \mathbb{R}$ is any nabla antidifference of $f$ on $\mathbb{N}_{a}^{b}$, that is $\nabla F(t)=f(t)$ for $t \in \mathbb{N}_{a+1}^{b}$. Then

$$
\int_{a}^{b} f(t) \nabla t=\left.F(t)\right|_{a} ^{b}:=F(b)-F(a) .
$$

To define the nabla exponential function we consider the set of regressive functions

$$
\mathcal{R}=\left\{p: \mathbb{N}_{a+1} \rightarrow \mathbb{R}: 1-p(t) \neq 0 \text { for } t \in \mathbb{N}_{a+1}\right\} .
$$

Then the nabla exponential function corresponding to $p \in \mathcal{R}$, based at $s \in \mathbb{N}_{a}$, is the unique solution $E_{p}(t, s)$ of the initial value problem

$$
\begin{gathered}
\nabla x(t)=p(t) x(t), \\
x(s)=1 .
\end{gathered}
$$

For the nabla exponential function, we have the following theorem. 
Theorem 1.1 (See [7, Theorem 3.6]). Assume $p \in \mathcal{R}$ and $s \in \mathbb{N}_{a}$. Then

$$
E_{p}(t, s)= \begin{cases}\prod_{j=s+1}^{t}(1-p(j))^{-1}, & t \in \mathbb{N}_{s} \\ \prod_{j=t+1}^{s}(1-p(j)), & t \in \mathbb{N}_{a}^{s-1}\end{cases}
$$

For any function $h$, it is understood that $\prod_{j=t+1}^{t} h(j)=1$.

For more background information on the nabla operator, we refer the reader to the monograph [7] by Goodrich and Peterson. Let

$$
\tau(t)=\min _{1 \leq i \leq n}\left\{\tau_{i}(t)\right\}, \quad t \in \mathbb{N}_{0}
$$

By a solution of the nabla difference equation (1.1), we mean a sequence of real numbers $x(t)$ for $t \geq \tau(0)$ which satisfy (1.1) for all $t \geq 0$. It is easy to see that (1.1) has a unique solution for any initial value conditions $x(t)=c(t), t \in \mathbb{N}_{\tau(0)}^{-1}$. As is customary, a nontrivial solution $x(t)$ of (1.1) is called oscillatory if for every $t_{0} \in \mathbb{N}_{0}$, there exists $t \geq t_{0}$ such that $x(t)=0$ or $x(t) x(t-1) \leq 0$. Otherwise, the solution is called non-oscillatory. This equation is called oscillatory if all its nontrivial solutions are oscillatory.

In the previous few decades, the oscillatory behavior of solutions to differential/delta difference equations has been extensively studied. See, for example, [1-6,8-14] and the references cited therein. However, none of these papers pays attention to the nabla difference equations. In the previous works about differential/delta difference equations, the authors consider conditions only related to largest delay argument or the smallest delay argument, as defined in (1.5); see, for example, [3-5]. However, existing research results show that the oscillatory behavior of the solutions of differential equations is related to all delay arguments, see [14]. Motivated by the above discussions, our objective is to establish oscillation criteria based on all the delay arguments of the nabla difference equation (1.1), as we did for differential equations in [14]. In our opinion, small changes in each delay will affect the oscillation of solutions to (1.1).

\section{Main Results}

Throughout this work, as usual, we assume that empty sums equal 0 . In addition, we use the conventions that $\left[t_{1}, t_{2}\right]=\left[t_{1}, t_{2}\right]_{\mathbb{R}} \cap \mathbb{N}_{0}$ and

$$
\mathbf{P}(t):=\sum_{i=1}^{n} p_{i}(t), \quad t \in \mathbb{N}_{0} .
$$


From (1.3), we are free to use the integral and summation symbols. According to the ideas in $[13,14]$, we define the discrete nabla multiple integral functions

$$
\begin{aligned}
\Phi_{\tau_{i}, p_{n_{1}}, p_{n_{2}}, \ldots, p_{n_{m}}}(t):= & \int_{\tau_{i}(t)}^{t} p_{n_{1}}\left(s_{1}\right) \int_{\tau_{n_{1}}\left(s_{1}\right)}^{\tau_{n_{1}}(t)} p_{n_{2}}\left(s_{2}\right) \cdots \\
& \int_{\tau_{n_{m-1}}\left(s_{m-1}\right)}^{\tau_{n_{m-1}}\left(\cdots \tau_{n_{1}}(t)\right)} p_{n_{m}}\left(s_{m}\right) \nabla s_{m} \cdots \nabla s_{2} \nabla s_{1},
\end{aligned}
$$

and

$$
\begin{aligned}
& \Psi_{\tau_{i}, p_{n_{1}}, p_{n_{2}}, \ldots, p_{n_{m}}}(t) \\
& :=\int_{\tau_{i}(t)}^{t} p_{n_{1}}\left(s_{1}\right) \int_{\tau_{n_{1}}\left(s_{1}\right)}^{\tau_{n_{1}}(t)} p_{n_{2}}\left(s_{2}\right) \cdots \int_{\tau_{n_{m-1}}\left(s_{m-1}\right)}^{\tau_{n_{m-1}}\left(\cdots \tau_{n_{1}}(t)\right)} p_{n_{m}}\left(s_{m}\right) \\
& \quad \times E_{\mathbf{P}}\left(\tau_{n_{m}}\left(\cdots\left(\tau_{n_{1}}(t)\right)\right), \tau_{n_{m}}\left(s_{m}\right)\right) \nabla s_{m} \cdots \nabla s_{2} \nabla s_{1}
\end{aligned}
$$

where $i, n_{1}, n_{2}, \ldots, n_{m} \in \mathbb{N}_{1}^{n}$ and $m \in \mathbb{N}_{1}$. Using (2.1) and (2.2), we then define the $n \times n$ matrix $A_{1}(t)$ with the entries

$$
a_{1 ; i n_{1}}(t)=\Psi_{\tau_{i}, p_{n_{1}}}(t), \quad i, n_{1} \in \mathbb{N}_{1}^{n},
$$

and the $n$ dimensional vector $\vec{F}_{1}=\left(F_{1 ; 1}, F_{1 ; 2}, \ldots, F_{1 ; n}\right)^{T}$ as the solution of the system of linear equations $\left(I-A_{1}\right) \vec{F}_{1}=\overrightarrow{1}=(1,1, \ldots, 1)^{T}$, when it exists. Using (2.1) and (2.2), we define the $n \times n$ matrix $A_{2}(t)$ with entries

$$
a_{2 ; i n_{1}}(t)=\Phi_{\tau_{i}, p_{n_{1}}}(t)+\sum_{n_{2}=1}^{n} \Psi_{\tau_{i}, p_{n_{1}}, p_{n_{2}}}(t) F_{1 ; n_{2}}\left(\tau_{n_{1}}(t)\right),
$$

where $i, n_{1} \in \mathbb{N}_{1}^{n}$, and the $n$ dimensional vector $\vec{F}_{2}=\left(F_{2 ; 1}, F_{2 ; 2}, \ldots, F_{2 ; n}\right)^{T}$ as the solution of the system of linear equations $\left(I-A_{2}\right) \vec{F}_{2}=\overrightarrow{1}$, when it exists. Recursively for $m \in \mathbb{N}_{3}$, we define the $n \times n$ matrices $A_{m}(t)$ with the entries

$$
\begin{aligned}
a_{m ; n_{1}}(t)= & \Phi_{\tau_{i}, p_{n_{1}}}(t)+\sum_{j=2}^{m-1} \sum_{n_{2}=1}^{n} \sum_{n_{3}=1}^{n} \cdots \sum_{n_{j}=1}^{n} \Phi_{\tau_{i}, p_{n_{1}}, \ldots, p_{n_{j}}}(t) \\
& \times \prod_{k=1}^{j-1} F_{m-k ; n_{1+k}}\left(\tau_{n_{k}} \cdots\left(\tau_{n_{1}}(t)\right)\right)+\sum_{n_{2}=1}^{n} \sum_{n_{3}=1}^{n} \cdots \\
& \sum_{n_{m}=1}^{n} \Psi_{\tau_{i}, p_{n_{1}}, \ldots, p_{n_{m}}}(t) \prod_{k=1}^{m-1} F_{m-k ; n_{1+k}}\left(\tau_{n_{k}} \cdots\left(\tau_{n_{1}}(t)\right)\right),
\end{aligned}
$$

where $i, n_{1} \in \mathbb{N}_{1}^{n}$. As before, we can define the vector

$$
\overrightarrow{F_{m}}=\left(F_{m ; 1}, F_{m ; 2}, \ldots, F_{m ; n}\right)^{T}
$$


as the solution to the system of linear equation

$$
\left(I-A_{m}\right) \overrightarrow{F_{m}}=\overrightarrow{1}=(1,1, \ldots, 1)^{T},
$$

and it will be used for finding a lower bound of $x\left(\tau_{i}(t)\right) / x(t)$ for positive solutions $x(t)$.

To prove our main results, we present several lemmas. Lemma 2.1 can be found in [14].

Lemma 2.1. Let $B$ be an $n \times n$ matrix with $b_{i j} \leq 0$ for $i \neq j, c_{i} \geq 1$ for $i \in \mathbb{N}_{1}^{n}$. If $y_{i}>0$ and $\vec{y}$ is a solution of $B \vec{y}=\vec{c}$, then $B$ is invertible, and $y_{i} \geq F_{i}>0$ for $i \in \mathbb{N}_{1}^{n}$, where $B \vec{F}=(1,1, \ldots, 1)^{T}$.

Lemma 2.2. Assume $x(t)$ is a solution of equation (1.1). Further, assume that there exit $m \geq 1$ and $t_{0}^{*} \geq t_{0} \geq 0$ such that $\tau^{m+2}\left(t_{0}\right) \geq 0$, and $p_{i}(t) \geq 0$ for $t \in\left[\tau^{m+1}\left(t_{0}\right), t_{0}^{*}\right]$ and $x(t)>0$ for $t \in\left[\tau^{m+2}\left(t_{0}\right), t_{0}^{*}\right]$. Then

$$
\frac{x\left(\tau_{i}(t)\right)}{x(t)} \geq F_{m ; i}(t) \quad \forall t \in\left[t_{0}, t_{0}^{*}\right]
$$

where $t_{0}, t_{0}^{*} \in \mathbb{N}_{0}, i \in \mathbb{N}_{1}^{n}, m \in \mathbb{N}_{1}, \tau(t)$ and $F_{m ; i}(t)$ are defined by (1.5) and (2.6) respectively.

Proof. Since $p_{i}(t) \geq 0$ and $x\left(\tau_{i}(t)>0\right.$ for $t \in\left[\tau^{m+1}\left(t_{0}\right), t_{0}^{*}\right]$, from (1.1), we have

$$
\nabla x(t)=-\sum_{i=1}^{n} p_{i}(t) x\left(\tau_{i}(t)\right) \leq 0, \quad t \in\left[\tau^{m+1}\left(t_{0}\right), t_{0}^{*}\right] .
$$

Therefore $x(t)$ is non-increasing. We have $x\left(\tau_{i}(t)\right) \geq x(t-1)$, since $\tau_{i}(t) \leq t-1$. From (2.8), we obtain

$$
x(t)-x(t-1) \leq-\sum_{i=1}^{n} p_{i}(t) x(t-1)
$$

which implies that

$$
x(t) \leq\left(1-\sum_{i=1}^{n} p_{i}(t)\right) x(t-1) .
$$

Therefore, for $s<t$, we have

$$
x(t) \leq \prod_{j=s+1}^{t}\left(1-\sum_{i=1}^{n} p_{i}(j)\right) x(s)
$$

that is,

$$
x(s) \geq x(t) \prod_{j=s+1}^{t}\left(1-\sum_{i=1}^{n} p_{i}(j)\right)^{-1}=x(t) E_{\mathbf{P}}(t, s) .
$$


Integrating (1.1) from $\tau_{i}(t)$ to $t$, by (1.4), we obtain

$$
x\left(\tau_{i}(t)\right)=x(t)+\sum_{n_{1}=1}^{n} \int_{\tau_{i}(t)}^{t} p_{n_{1}}\left(s_{1}\right) x\left(\tau_{n_{1}}\left(s_{1}\right)\right) \nabla s_{1}, \quad i \in \mathbb{N}_{1}^{n} .
$$

Since $s_{1} \leq t$ and $\tau_{n_{1}}$ is non-decreasing, we estimate $x\left(\tau_{n_{1}}\left(s_{1}\right)\right)$ by integrating (1.1) from $\tau_{n_{1}}\left(s_{1}\right)$ to $\tau_{n_{1}}(t)$; so that by (1.4), we obtain

$$
x\left(\tau_{n_{1}}\left(s_{1}\right)\right)=x\left(\tau_{n_{1}}(t)\right)+\sum_{n_{2}=1}^{n} \int_{\tau_{n_{1}}\left(s_{1}\right)}^{\tau_{n_{1}}(t)} p_{n_{2}}\left(s_{2}\right) x\left(\tau_{n_{2}}\left(s_{2}\right)\right) \nabla s_{2}, \quad n_{1} \in \mathbb{N}_{1}^{n} .
$$

From (2.10) and (2.11), it is easy to see that

$$
\begin{aligned}
x\left(\tau_{i}(t)\right)= & x(t)+\sum_{n_{1}=1}^{n} \int_{\tau_{i}(t)}^{t} p_{n_{1}}\left(s_{1}\right) \\
& \times\left[x\left(\tau_{n_{1}}(t)\right)+\sum_{n_{2}=1}^{n} \int_{\tau_{n_{1}}\left(s_{1}\right)}^{\tau_{n_{1}}(t)} p_{n_{2}}\left(s_{2}\right) x\left(\tau_{n_{2}}\left(s_{2}\right)\right) \nabla s_{2}\right] \nabla s_{1} .
\end{aligned}
$$

Integrating (1.1) from $\tau_{n_{2}}\left(s_{2}\right)$ to $\tau_{n_{2}}\left(\tau_{n_{1}}(t)\right)$, by (1.4), for $n_{2} \in \mathbb{N}_{1}^{n}$, we have

$$
x\left(\tau_{n_{2}}\left(s_{2}\right)\right)=x\left(\tau_{n_{2}}\left(\tau_{n_{1}}(t)\right)\right)+\sum_{n_{3}=1}^{n} \int_{\tau_{n_{2}}\left(s_{2}\right)}^{\tau_{n_{2}}\left(\tau_{n_{1}}(t)\right)} p_{n_{3}}\left(s_{3}\right) x\left(\tau_{n_{3}}\left(s_{3}\right)\right) \nabla s_{3} .
$$

From (2.12) and (2.13), we immediately obtain

$$
\begin{gathered}
x\left(\tau_{i}(t)\right)=x(t)+\sum_{n_{1}=1}^{n} \int_{\tau_{i}(t)}^{t} p_{n_{1}}\left(s_{1}\right)\left[x\left(\tau_{n_{1}}(t)\right)+\sum_{n_{2}=1}^{n} \int_{\tau_{n_{1}}\left(s_{1}\right)}^{\tau_{n_{1}}(t)} p_{n_{2}}\left(s_{2}\right)\right. \\
\left.\left[x\left(\tau_{n_{2}}\left(\tau_{n_{1}}(t)\right)\right)+\sum_{n_{3}=1}^{n} \int_{\tau_{n_{2}}\left(s_{2}\right)}^{\tau_{n_{2}}\left(\tau_{n_{1}}(t)\right)} p_{n_{3}}\left(s_{3}\right) x\left(\tau_{n_{3}}\left(s_{3}\right)\right) \nabla s_{3}\right] \nabla s_{2}\right] \nabla s_{1} .
\end{gathered}
$$

For $t \in\left[\tau^{m-1}\left(t_{0}\right), t_{0}^{*}\right]$, from (2.9), for $s_{1}$ as in (2.10), we have

$$
\begin{aligned}
x\left(\tau_{n_{1}}\left(s_{1}\right)\right) & \geq x\left(\tau_{n_{1}}(t)\right) \prod_{j=\tau_{n_{1}}\left(s_{1}\right)+1}^{\tau_{n_{1}}(t)}\left(1-\sum_{i=1}^{n} p_{i}(j)\right)^{-1} \\
& =x\left(\tau_{n_{1}}(t)\right) E_{\mathbf{P}}\left(\tau_{n_{1}}(t), \tau_{n_{1}}\left(s_{1}\right)\right) .
\end{aligned}
$$

From (2.1), (2.10) and (2.15), it follows that

$$
x\left(\tau_{i}(t)\right) \geq x(t)+\sum_{n_{1}=1}^{n} x\left(\tau_{n_{1}}(t)\right) \int_{\tau_{i}(t)}^{t} p_{n_{1}}\left(s_{1}\right) E_{\mathbf{P}}\left(\tau_{n_{1}}(t), \tau_{n_{1}}\left(s_{1}\right)\right) \nabla s_{1} .
$$


From (2.2), we immediately obtain

$$
x\left(\tau_{i}(t)\right) \geq x(t)+\sum_{n_{1}=1}^{n} x\left(\tau_{n_{1}}(t)\right) \Psi_{\tau_{i}, p_{n_{1}}}(t),
$$

where $i \in \mathbb{N}_{1}^{n}$. Dividing both sides of the above inequality by $x(t)$, we have $\vec{y} \geq \overrightarrow{1}+A_{1} \vec{y}$ that is $\left(I-A_{1}\right) \vec{y} \geq \overrightarrow{1}$, where $y_{i}=x\left(\tau_{i}(t)\right) / x(t)$ and the matrix $A_{1}(t)$ with the entries $a_{1 ; i n_{1}}(t)=\Psi_{\tau_{i}, p_{n_{1}}}(t), i, n_{1} \in \mathbb{N}_{1}^{n}$. Using Lemma 2.1 with $B=I-A_{1}$, we obtain that

$$
\frac{x\left(\tau_{i}(t)\right)}{x(t)} \geq F_{1 ; i}(t)>0, \quad t \in\left[\tau^{m-1}\left(t_{0}\right), t_{0}^{*}\right], \quad i \in \mathbb{N}_{1}^{n},
$$

where $\vec{F}_{1}$ satisfies $\left(I-A_{1}\right) \vec{F}_{1}=\overrightarrow{1}$. For $t \in\left[\tau^{m-2}\left(t_{0}\right), t_{0}^{*}\right]$, from (2.9) and (2.16), we have

$$
\begin{aligned}
x\left(\tau_{n_{2}}\left(s_{2}\right)\right) & \geq x\left(\tau_{n_{2}}\left(\tau_{n_{1}}(t)\right)\right) E_{\mathbf{P}}\left(\tau_{n_{2}}\left(\tau_{n_{1}}(t)\right), \tau_{n_{2}}\left(s_{2}\right)\right) \\
& \geq F_{1 ; n_{2}}\left(\tau_{n_{1}}(t)\right) x\left(\tau_{n_{1}}(t) E_{\mathbf{P}}\left(\tau_{n_{2}}\left(\tau_{n_{1}}(t)\right), \tau_{n_{2}}\left(s_{2}\right)\right) .\right.
\end{aligned}
$$

From (2.1), (2.2) and(2.12), it follows that

$$
\begin{aligned}
x\left(\tau_{i}(t)\right) \geq & x(t)+\sum_{n_{1}=1}^{n} x\left(\tau_{n_{1}}(t)\right) \int_{\tau_{i}(t)}^{t} p_{n_{1}}\left(s_{1}\right) \nabla s_{1}+\sum_{n_{1}=1}^{n} x\left(\tau_{n_{1}}(t)\right) \int_{\tau_{i}(t)}^{t} p_{n_{1}}\left(s_{1}\right) \\
& \times \sum_{n_{2}=1}^{n} F_{1 ; n_{2}}\left(\tau_{n_{1}}(t)\right) \int_{\tau_{n_{1}}\left(s_{1}\right)}^{\tau_{n_{1}}(t)} p_{n_{2}}\left(s_{2}\right) E_{\mathbf{P}}\left(\tau_{n_{2}}\left(\tau_{n_{1}}(t)\right), \tau_{n_{2}}\left(s_{2}\right)\right) \nabla s_{2} \nabla s_{1} \\
= & x(t)+\sum_{n_{1}=1}^{n} x\left(\tau_{n_{1}}(t)\right)\left[\Phi_{\tau_{i}, p_{n_{1}}}(t)+\sum_{n_{2}=1}^{n} \Psi_{\tau_{i}, p_{n_{1}}, p_{n_{2}}}(t) F_{1 ; n_{2}}\left(\tau_{n_{1}}(t)\right)\right],
\end{aligned}
$$

From the above inequality, we define the matrix $A_{2}(t)$ with entries

$$
a_{2 ; i n_{1}}(t)=\Phi_{\tau_{i}, p_{n_{1}}}(t)+\sum_{n_{2}=1}^{n} \Psi_{\tau_{i}, p_{n_{1}}, p_{n_{2}}}(t) F_{1 ; n_{2}}\left(\tau_{n_{1}}(t)\right),
$$

where $i, n_{1} \in \mathbb{N}_{1}^{n}$. Let $y_{i}=x\left(\tau_{i}(t)\right) / x(t)$. Using Lemma 2.1 with $B=I-A_{2}$, we obtain

$$
\frac{x\left(\tau_{i}(t)\right)}{x(t)} \geq F_{2 ; i}(t)>0, \quad t \in\left[\tau^{m-2}\left(t_{0}\right), t_{0}^{*}\right], i \in \mathbb{N}_{1}^{n},
$$

where $\left(I-A_{2}\right) \vec{F}_{2}=\overrightarrow{1}$. For $t \in\left[\tau^{m-3}\left(t_{0}\right), t_{0}^{*}\right]$, from (2.9), (2.16) and (2.17), we have

$$
\begin{aligned}
& x\left(\tau_{n_{3}}\left(s_{3}\right)\right) \\
& \geq x\left(\tau_{n_{3}}\left(\tau_{n_{2}}\left(\tau_{n_{1}}(t)\right)\right)\right) E_{\mathbf{P}}\left(\tau_{n_{3}}\left(\tau_{n_{2}}\left(\tau_{n_{1}}(t)\right)\right), \tau_{n_{3}}\left(s_{3}\right)\right) \\
& \geq F_{1 ; n_{3}}\left(\tau_{n_{2}}\left(\tau_{n_{1}}(t)\right)\right) F_{2 ; n_{2}}\left(\tau_{n_{1}}(t)\right) x\left(\tau_{n_{1}}(t)\right) E_{\mathbf{P}}\left(\tau_{n_{3}}\left(\tau_{n_{2}}\left(\tau_{n_{1}}(t)\right)\right), \tau_{n_{3}}\left(s_{3}\right)\right) .
\end{aligned}
$$


From (2.1), (2.2) and(2.14), it follows that

$$
\begin{aligned}
x\left(\tau_{i}(t)\right) \geq & x(t)+\sum_{n_{1}=1}^{n} x\left(\tau_{n_{1}}(t)\right) \int_{\tau_{i}(t)}^{t} p_{n_{1}}\left(s_{1}\right) \nabla s_{1} \\
& +\sum_{n_{1}=1}^{n} x\left(\tau_{n_{1}}(t)\right) \sum_{n_{2}=1}^{n} \int_{\tau_{i}(t)}^{t} p_{n_{1}}\left(s_{1}\right) \int_{\tau_{n_{1}}\left(s_{1}\right)}^{\tau_{n_{1}}(t)} p_{n_{2}}\left(s_{2}\right) F_{2 ; n_{2}}\left(\tau_{n_{1}}(t)\right) \nabla s_{2} \nabla s_{1} \\
& +\sum_{n_{1}=1}^{n} x\left(\tau_{n_{1}}(t)\right) \sum_{n_{2}=1}^{n} F_{2 ; n_{2}}\left(\tau_{n_{1}}(t)\right) \sum_{n_{3}=1}^{n} F_{1 ; n_{3}}\left(\tau_{n_{2}}\left(\tau_{n_{1}}(t)\right)\right) \\
& \times \int_{\tau_{i}(t)}^{t} p_{n_{1}}\left(s_{1}\right) \int_{\tau_{n_{1}}\left(s_{1}\right)}^{\tau_{n_{1}}(t)} p_{n_{2}}\left(s_{2}\right) \int_{\tau_{n_{2}}\left(s_{2}\right)}^{\tau_{n_{2}}\left(\tau_{n_{1}}(t)\right)} p_{n_{3}}\left(s_{3}\right) \\
& \times E_{\mathbf{P}}\left(\tau_{n_{3}}\left(\tau_{n_{2}}\left(\tau_{n_{1}}(t)\right)\right), \tau_{n_{3}}\left(s_{3}\right)\right) \nabla s_{3} \nabla s_{2} \nabla s_{1} \\
= & x(t)+\sum_{n_{1}=1}^{n} x\left(\tau_{n_{1}}(t)\right)\left[\Phi_{\tau_{i}, p_{n_{1}}}(t)+\sum_{n_{2}=1}^{n} \Phi_{\tau_{i}, p_{n_{1}}, p_{n_{2}}}(t) F_{1 ; n_{2}}\left(\tau_{n_{1}}(t)\right)\right. \\
& \left.+\sum_{n_{2}=1}^{n} \sum_{n_{3}=1}^{n} \Psi_{\tau_{i}, p_{n_{1}}, p_{n_{2}}, p_{n_{3}}}(t) F_{1 ; n_{3}}\left(\tau_{n_{2}}\left(\tau_{n_{1}}(t)\right)\right) F_{2 ; n_{2}}\left(\tau_{n_{1}}(t)\right)\right] .
\end{aligned}
$$

Let $A_{3}(t)$ be the matrix with entries

$$
\begin{aligned}
a_{3 ; n_{1}}(t)= & \Phi_{\tau_{i}, p_{n_{1}}}(t)+\sum_{n_{2}=1}^{n} \Phi_{\tau_{i}, p_{n_{1}}, p_{n_{2}}}(t) F_{1 ; n_{2}}\left(\tau_{n_{1}}(t)\right) \\
& +\sum_{n_{2}=1}^{n} \sum_{n_{3}=1}^{n} \Psi_{\tau_{i}, p_{n_{1}}, p_{n_{2}}, p_{n_{3}}}(t) F_{1 ; n_{3}}\left(\tau_{n_{2}}\left(\tau_{n_{1}}(t)\right)\right) F_{2 ; n_{2}}\left(\tau_{n_{1}}(t)\right),
\end{aligned}
$$

where $i, n_{1} \in \mathbb{N}_{1}^{n}$. Using Lemma 2.1 with $B=I-A_{3}$, we have

$$
\frac{x\left(\tau_{i}(t)\right)}{x(t)} \geq F_{3 ; i}(t)>0, \quad\left[\tau^{m-3}\left(t_{0}\right), t_{0}^{*}\right], i \in \mathbb{N}_{1}^{n}
$$

where $\left(I-A_{3}\right) \vec{F}_{3}=\overrightarrow{1}$.

Following the same procedure $m$ times, it is easy to obtain the statement of Lemma 2.2. This completes the proof.

Theorem 2.3. Assume that there exists a positive integer $m$, such that $\tau^{m+2}\left(t_{0}\right) \geq 0$, and $p_{i}(t) \geq 0$ for $t \in\left[\tau^{m+1}\left(t_{0}\right), t_{0}\right]$. If there exists $i \in \mathbb{N}_{1}^{n}$ such that $F_{1 ; i}\left(t_{0}\right)>0$, $F_{2 ; i}\left(t_{0}\right)>0, \ldots, F_{m-1 ; i}\left(t_{0}\right)>0$, and $F_{m ; i}\left(t_{0}\right)$ is not defined or is negative, where $F_{m ; i}(t)$ is defined by (2.6). Then for any $t \geq t_{0}$, every solution of equation (1.1) has at least one zero on $\left[\tau^{m+2}\left(t_{0}\right), t_{0}\right]$. 
Proof. Suppose that $x(t)$ is a solution of equation (1.1). Without loss of generality, we may assume that $x(t)>0$ for $t \in\left[\tau^{m+2}\left(t_{0}\right), t_{0}\right]$. We shall only consider this case, since the substitution $z(t)=-x(t)$ transforms (1.1) into an equation of the same form. From Lemma 2.2 with $t_{0}=t_{0}^{*}$, we obtain $F_{m, i}\left(t_{0}\right)>0$ for $i \in \mathbb{N}_{1}^{n}$, which is a contradiction to our hypothesis. This completes the proof of Theorem 2.3.

By repeatedly Using Lemma 2.2 to some increasing sequences $\left\{t_{k}\right\}_{k=1}^{\infty}$ approaching $\infty$, one can prove that the following theorem in a manner similar to that of Theorem 2.3. The details of the proof are therefore are omitted.

Theorem 2.4. Suppose that there exist some increasing sequences $\left\{t_{k}\right\}_{k=1}^{\infty}$ approaching $\infty$ and some bounded sequences of positive integers $\left\{m_{k}\right\}_{k=1}^{\infty}$, such that $\tau^{m_{k}+2}\left(t_{k}\right) \geq 0$ for each $k$, and $p_{i}(t) \geq 0$ for $t \in\left[\tau^{m_{k}+1}\left(t_{k}\right), t_{k}\right]$. If for each $t_{k}$, there exists $i \in \mathbb{N}_{1}^{n}$ such that $F_{1 ; i}\left(t_{k}\right)>0, F_{2 ; i}\left(t_{k}\right)>0, \ldots, F_{m_{k}-1 ; i}\left(t_{k}\right)>0$, and $F_{m_{k} ; i}\left(t_{k}\right)$ is not defined or is negative. Then equation (1.1) is oscillatory.

\section{Examples}

In this section, we present some numerical examples that illustrate the main results of this paper. We first consider the first-order linear nabla difference equation with two constant delays,

$$
\nabla x(t)+p_{1} x\left(t-\tau_{1}\right)+p_{2} x\left(t-\tau_{2}\right)=0, \quad t \in \mathbb{N}_{0},
$$

where $p_{1}, p_{2}, \tau_{1}, \tau_{2}$ are positive constants.

Lemma 3.1. If $p_{i}(t) \equiv p_{i}>0$ and $\tau_{i}(t) \equiv t-\tau_{i}$ satisfying $\tau_{i}>0$, then for $i, n_{1}, \ldots, n_{m}$ in $\mathbb{N}_{1}^{n}$ and $m \in \mathbb{N}_{1}$,

$$
\Phi_{\tau_{i}, p_{n_{1}}, p_{n_{2}}, \ldots, p_{n_{m}}}(t)=\frac{1}{m !}\left(\tau_{i}-(m-1)\right)^{\bar{m}} \prod_{j=1}^{m} p_{n_{j}}=\left(\begin{array}{c}
\tau_{i} \\
m
\end{array}\right) \prod_{j=1}^{m} p_{n_{j}} .
$$

Proof. Since $p_{i}(t) \equiv p_{i}>0$ and $\tau_{i}(t) \equiv t-\tau_{i}$ satisfying $\tau_{i}>0$, from the definition of the nabla operator and the fundamental theorem for the nabla calculus, it is easy to see that

$$
\begin{aligned}
\int_{\tau_{n_{m-1}}\left(s_{m-1}\right)}^{\tau_{n_{m-1}}\left(\cdots \tau_{n_{1}}(t)\right)} p_{n_{m}}\left(s_{m}\right) \nabla s_{m} & =\int_{s_{m-1}-\tau_{n_{m-1}}}^{t-\tau_{n_{1}}-\cdots-\tau_{n_{m-1}}} p_{n_{m}} \nabla s_{m} \\
& =p_{n_{m}}\left[s_{m}{ }^{1}\right]_{s_{m-1}-\tau_{n_{m-1}}}^{t-\tau_{n_{1}}-\cdots-\tau_{n_{m-1}}} \\
& =p_{n_{m}}\left(t-\tau_{n_{1}}-\cdots-\tau_{n_{m-2}}-s_{m-1}\right)^{\overline{1}}
\end{aligned}
$$


From (1.2) and (1.4), it follows that

$$
\begin{aligned}
& \int_{\tau_{n_{m-2}}\left(s_{m-2}\right)}^{\tau_{n_{m-2}}\left(\cdots \tau_{n_{1}}(t)\right)} p_{n_{m-1}}\left(s_{m-1}\right) \int_{\tau_{n_{m-1}}\left(s_{m-1}\right)}^{\tau_{n_{m-1}}\left(\cdots \tau_{n_{1}}(t)\right)} p_{n_{m}}\left(s_{m}\right) \nabla s_{m} \nabla s_{m-1} \\
& =p_{n_{m-1}} p_{n_{m}} \int_{s_{m-2}-\tau_{n_{m-2}}}^{t-\tau_{n_{1}}-\cdots-\tau_{n_{m-2}}}\left(t-\tau_{n_{1}}-\cdots-\tau_{n_{m-2}}-s_{m-1}\right)^{\overline{1}} \nabla s_{m-1} \\
& =p_{n_{m-1}} p_{n_{m}}\left[-\frac{1}{2 !}\left(t-\tau_{n_{1}}-\cdots-\tau_{n_{m-2}}-\left(s_{m-1}+1\right)\right)^{\overline{2}}\right]_{s_{m-2}-\tau_{n_{m-2}}}^{t-\tau_{n_{1}}-\cdots-\tau_{n_{m-2}}} \\
& =p_{n_{m-1}} p_{n_{m}} \frac{1}{2 !}\left(t-\tau_{n_{1}}-\cdots-\tau_{n_{m-3}}-s_{m-2}-1\right)^{\overline{2}}
\end{aligned}
$$

In a manner similar to the above process, for $i, n_{1}, \cdots, n_{m} \in \mathbb{N}_{1}^{n}$ and $m \in \mathbb{N}_{1}$ we obtain

$$
\begin{aligned}
\Phi_{\tau_{i}, p_{n_{1}}, p_{n_{2}}, \ldots, p_{n_{m}}}(t) & =\prod_{j=1}^{m} p_{n_{j}} \frac{1}{(m-1) !} \int_{t-\tau_{i}}^{t}\left(t-(m-2)-s_{1}\right)^{\overline{m-1}} \nabla s_{1} \\
& =-\prod_{j=1}^{m} p_{n_{j}} \frac{1}{m !}\left(\left(t-(m-1)-s_{1}\right)^{\bar{m}}\right)_{t-\tau_{i}}^{t} \\
& =\frac{1}{m !}\left(\tau_{i}-(m-1)\right)^{\bar{m}} \prod_{j=1}^{m} p_{n_{j}} \\
& =\left(\begin{array}{c}
\tau_{i} \\
m
\end{array}\right) \prod_{j=1}^{m} p_{n_{j}}
\end{aligned}
$$

which completes the proof.

Lemma 3.2. If $p_{i}(t) \equiv p_{i}>0$ and $\tau_{i}(t) \equiv t-\tau_{i}$ satisfying $\tau_{i}>0$, then for $i, n_{1}, \ldots, n_{m}$ in $\mathbb{N}_{1}^{n}$ and $m \in \mathbb{N}_{1}$

$$
\Psi_{\tau_{i}, p_{n_{1}}, p_{n_{2}}, \ldots, p_{n_{m}}}=p_{n_{m}} \frac{1-\mathbf{P}}{\mathbf{P}}\left(\Psi_{\tau_{i}, p_{n_{1}}, p_{n_{2}}, \ldots, p_{n_{(m-1)}}}-\Phi_{\tau_{i}, p_{n_{1}}, p_{n_{2}}, \ldots, p_{n_{(m-1)}}}\right),
$$

where $\mathbf{P}=\sum_{k=1}^{n} p_{k}, \Phi_{\tau_{i}, p_{n_{1}}, p_{n_{2}}, \ldots, p_{n_{m}}}$ is given by Lemma 3.1 and

$$
\Psi_{\tau_{i}, p_{n_{1}}}=\frac{p_{n_{1}}\left(1-(1-\mathbf{P})^{\tau_{i}}\right)}{\mathbf{P}(1-\mathbf{P})^{\tau_{i}-1}}
$$

Proof. Since $p_{i}(t) \equiv p_{i}>0$ and $\tau_{i}(t) \equiv t-\tau_{i}$ satisfying $\tau_{i}>0$, from the definitions of the nabla operator and the nabla exponential function, it is easy to see that

$$
\nabla_{s} E_{\mathbf{P}}(t, s)=-\frac{\mathbf{P}}{1-\mathbf{P}} E_{\mathbf{P}}(t, s)
$$


From the fundamental theorem for the nabla calculus, it follows that

$$
\begin{aligned}
\Psi_{\tau_{i}, p_{n_{1}}} & =\int_{t-\tau_{i}}^{t} p_{n_{1}} E_{\mathbf{P}}\left(t-\tau_{n_{1}}, s_{1}-\tau_{n_{1}}\right) \nabla s_{1} \\
& =p_{n_{1}} \frac{1-\mathbf{P}}{\mathbf{P}}\left((1-\mathbf{P})^{-\tau_{i}}-1\right)=\frac{p_{n_{1}}\left(1-(1-\mathbf{P})^{\tau_{i}}\right)}{\mathbf{P}(1-\mathbf{P})^{\tau_{i}-1}}, \\
\Psi_{\tau_{i}, p_{n_{1}}, p_{n_{2}}} & =\int_{t-\tau_{i}}^{t} p_{n_{1}} \int_{s_{1}-\tau_{n_{1}}}^{t-\tau_{n_{1}}} p_{n_{2}} E_{\mathbf{P}}\left(t-\tau_{n_{1}}-\tau_{n_{2}}, s_{2}-\tau_{n_{2}}\right) \nabla s_{2} \nabla s_{1} \\
& =p_{n_{2}} \frac{1-\mathbf{P}}{\mathbf{P}}\left(\Psi_{\tau_{i}, p_{n_{1}}}-\Phi_{\tau_{i}, p_{n_{1}}}\right) .
\end{aligned}
$$

In general, we obtain that

$$
\Psi_{\tau_{i}, p_{n_{1}}, p_{n_{2}}, \ldots, p_{n_{m}}}=p_{n_{m}} \frac{1-\mathbf{P}}{\mathbf{P}}\left(\Psi_{\tau_{i}, p_{n_{1}}, p_{n_{2}}, \ldots, p_{n_{(m-1)}}}-\Phi_{\tau_{i}, p_{n_{1}}, p_{n_{2}}, \ldots, p_{n_{(m-1)}}}\right),
$$

where $i, n_{1}, \cdots, n_{m} \in \mathbb{N}_{1}^{n}, m \in \mathbb{N}_{1}$. The proof is complete.

Using (2.1), (2.2), Lemmas 3.1 and 3.2, we have

$$
\Phi_{\tau_{i}, p_{n_{1}}, p_{n_{2}}, \ldots, p_{n_{m}}}= \begin{cases}p_{1}{ }^{k} p_{2}{ }^{m-k}\left(\begin{array}{c}
\tau_{i} \\
m
\end{array}\right), & 1 \leq m \leq \tau_{i} \\
0, & m>\tau_{i},\end{cases}
$$

while for $1 \leq m \leq \tau_{i}$,

$$
\Psi_{\tau_{i}, p_{n_{1}}, p_{n_{2}}, \ldots, p_{n_{m}}}=p_{n_{m}} \frac{1-p_{1}-p_{2}}{p_{1}+p_{2}}\left(\Psi_{\tau_{i}, p_{n_{1}}, \ldots, p_{n_{(m-1)}}}-\Phi_{\tau_{i}, p_{n_{1}}, \ldots, p_{n_{(m-1)}}}\right)
$$

and

$$
\Psi_{\tau_{i}, p_{n_{1}}, p_{n_{2}}, \ldots, p_{n_{m}}}=0, \quad m>\tau_{i},
$$

where $i, n_{1}, \cdots, n_{m} \in \mathbb{N}_{1}^{2}, m \in \mathbb{N}_{1}, k$ is the number of occurrences of $p_{1}$, and

$$
\Psi_{\tau_{i}, p_{n_{1}}}=\frac{p_{n_{1}}\left(1-\left(1-p_{1}-p_{2}\right)^{\tau_{i}}\right)}{\left(p_{1}+p_{2}\right)\left(1-p_{1}-p_{2}\right)^{\tau_{i}-1}} .
$$

From (3.2) and (3.3), we define the matrix $A_{1}$ with entries

$$
a_{1 ; i n_{1}}=\Psi_{\tau_{i}, p_{n_{1}}}=\frac{p_{n_{1}}\left(1-\left(1-p_{1}-p_{2}\right)^{\tau_{i}}\right)}{\left(p_{1}+p_{2}\right)\left(1-p_{1}-p_{2}\right)^{\tau_{i}-1}}
$$

where $i, n_{1}=1,2$. Then by Lemma 2.2 with $B=I-A_{1}$, the lower bounds are

$$
F_{1 ; 1}=\frac{1-a_{1 ; 22}+a_{1 ; 12}}{\left(1-a_{1 ; 11}\right)\left(1-a_{1 ; 22}\right)-a_{1 ; 12} a_{1 ; 21}},
$$




$$
F_{1 ; 2}=\frac{1-a_{1 ; 11}+a_{1 ; 21}}{\left(1-a_{1 ; 11}\right)\left(1-a_{1 ; 22}\right)-a_{1 ; 12} a_{1 ; 21}} .
$$

The matrix $A_{2}$ has entries

$$
\begin{aligned}
a_{2 ; i n_{1}} & =\Phi_{\tau_{i}, p_{n_{1}}}+\sum_{n_{2}=1}^{n} \Psi_{\tau_{i}, p_{n_{1}}, p_{n_{2}}} F_{1 ; n_{2}} \\
& =\Phi_{\tau_{i}, p_{n_{1}}}+\sum_{n_{2}=1}^{n} p_{n_{2}} F_{1 ; n_{2}} \frac{1-p_{1}-p_{2}}{p_{1}+p_{2}}\left(\Psi_{\tau_{i}, p_{n_{1}}}-\Phi_{\tau_{i}, p_{n_{1}}}\right),
\end{aligned}
$$

where $i, n_{1}, n_{2}=1,2$. From (2.5), for $m \geq 3$, the matrix $A_{m}$ has entries

$$
\begin{aligned}
a_{m ; n_{1}}= & p_{n_{1}} \sum_{j=1}^{m-1}\left(\begin{array}{c}
\tau_{i} \\
j
\end{array}\right) \prod_{k=1}^{j-1}\left(p_{1} F_{m-k ; 1}+p_{2} F_{m-k ; 2}\right)+\sum_{n_{2}=1}^{n} \sum_{n_{3}=1}^{n} \cdots \\
& \sum_{n_{m}=1}^{n} \prod_{j=1}^{m} p_{n_{j}}\left(\frac{\left(1-\left(1-p_{1}-p_{2}\right)^{\tau_{i}}\right)}{\left(p_{1}+p_{2}\right)^{m}\left(1-p_{1}-p_{2}\right)^{\tau_{i}-m}}\right. \\
& \left.-\sum_{j=2}^{m}\left(\begin{array}{c}
\tau_{i} \\
j-1
\end{array}\right)\left(\frac{1-p_{1}-p_{2}}{p_{1}+p_{2}}\right)^{m-j+1}\right) \prod_{k=1}^{m-1} F_{m-k ; n_{1+k}}
\end{aligned}
$$

where $i, n_{1}, n_{2}, \ldots, n_{m}=1,2$. By Lemma 2.2, it follows that

$$
\begin{aligned}
& F_{m: 1}=\frac{1-a_{m ; 22}+a_{m ; 12}}{\left(1-a_{m ; 11}\right)\left(1-a_{m ; 22}\right)-a_{m ; 12} a_{m ; 21}}, \\
& F_{m ; 2}=\frac{1-a_{m ; 11}+a_{m ; 21}}{\left(1-a_{m ; 11}\right)\left(1-a_{m ; 22}\right)-a_{m ; 12} a_{m ; 21}} .
\end{aligned}
$$

Taking $\tau_{1}=2$ and $\tau_{2}=3$, from (3.2), (3.3) and (3.4), we obtain

$$
\begin{aligned}
& a_{1 ; 11}=\frac{p_{1}\left(1-\left(1-p_{1}-p_{2}\right)^{2}\right)}{\left(p_{1}+p_{2}\right)\left(1-p_{1}-p_{2}\right)}, \quad a_{1 ; 12}=\frac{p_{2}\left(1-\left(1-p_{1}-p_{2}\right)^{2}\right)}{\left(p_{1}+p_{2}\right)\left(1-p_{1}-p_{2}\right)}, \\
& a_{1 ; 21}=\frac{p_{1}\left(1-\left(1-p_{1}-p_{2}\right)^{3}\right)}{\left(p_{1}+p_{2}\right)\left(1-p_{1}-p_{2}\right)^{2}}, \quad a_{1 ; 22}=\frac{p_{2}\left(1-\left(1-p_{1}-p_{2}\right)^{3}\right)}{\left(p_{1}+p_{2}\right)\left(1-p_{1}-p_{2}\right)^{2}} ; \\
& a_{2 ; 11}=2 p_{1}+p_{1}\left(p_{1} F_{1 ; 1}+p_{2} F_{1 ; 2}\right), \quad a_{2 ; 12}=2 p_{2}+p_{2}\left(p_{1} F_{1 ; 1}+p_{2} F_{1 ; 2}\right), \\
& a_{2 ; 21}=3 p_{1}+\frac{p_{1}\left(3-2\left(p_{1}+p_{2}\right)\right)\left(p_{1} F_{1 ; 1}+p_{2} F_{1 ; 2}\right)}{1-p_{1}-p_{2}}, \\
& a_{2 ; 22}=3 p_{2}+\frac{p_{2}\left(3-2\left(p_{1}+p_{2}\right)\right)\left(p_{1} F_{1 ; 1}+p_{2} F_{1 ; 2}\right)}{1-p_{1}-p_{2}} ; \\
& a_{3 ; 11}=2 p_{1}+p_{1}\left(p_{1} F_{2 ; 1}+p_{2} F_{2 ; 2}\right), \quad a_{3 ; 12}=2 p_{2}+p_{2}\left(p_{1} F_{2 ; 1}+p_{2} F_{2 ; 2}\right), \\
& a_{3 ; 21}=3 p_{1}+3 p_{1}\left(p_{1} F_{2 ; 1}+p_{2} F_{2 ; 2}\right)+p_{1} \prod_{k=1}^{2}\left(p_{1} F_{3-k ; 1}+p_{2} F_{3-k ; 2}\right),
\end{aligned}
$$




$$
a_{3 ; 22}=3 p_{2}+3 p_{2}\left(p_{1} F_{2 ; 1}+p_{2} F_{2 ; 2}\right)+p_{2} \prod_{k=1}^{2}\left(p_{1} F_{3-k ; 1}+p_{2} F_{3-k ; 2}\right) .
$$

In general, for $m \geq 4$, we have

$$
\begin{aligned}
& a_{m ; 11}=2 p_{1}+p_{1}\left(p_{1} F_{m-1 ; 1}+p_{2} F_{m-1 ; 2}\right), \\
& a_{m ; 12}=2 p_{2}+p_{2}\left(p_{1} F_{m-1 ; 1}+p_{2} F_{m-1 ; 2}\right), \\
& a_{m ; 21}=3 p_{1}+3 p_{1}\left(p_{1} F_{m-1 ; 1}+p_{2} F_{m-1 ; 2}\right)+p_{1} \prod_{k=1}^{2}\left(p_{1} F_{3-k ; 1}+p_{2} F_{3-k ; 2}\right), \\
& a_{m ; 22}=3 p_{2}+3 p_{2}\left(p_{1} F_{m-1 ; 1}+p_{2} F_{m-1 ; 2}\right)+p_{2} \prod_{k=1}^{2}\left(p_{1} F_{3-k ; 1}+p_{2} F_{3-k ; 2}\right), \\
& F_{m: 1}=\frac{1-a_{m ; 22}+a_{m ; 12}}{1-a_{m ; 11}-a_{m ; 22}}, \quad F_{m ; 2}=\frac{1-a_{m ; 11}+a_{m ; 21}}{1-a_{m ; 11}-a_{m ; 22}} .
\end{aligned}
$$

We note that $F_{m ; 1}$ and $F_{m ; 2}$ may not be defined or be negative. Some detailed data can be found in Table 3.1 in the Appendix. From Theorem 2.4, one can obtain the following corollary.

Corollary 3.3. If there exists an integer $m^{*} \geq 1$ such that $F_{1 ; i}>0, F_{2 ; i}>0, \ldots$, $F_{m^{*}-1 ; i}>0$, and $F_{m^{*} ; i}$ not defined or negative for some $i \in\{1,2\}$, where $F_{m^{*} ; i}$ is given by (3.4), then equation (3.1) is oscillatory.

Here, we give some data results for some simple cases. we only consider equation (3.1) with $\tau_{1}=2$ and $\tau_{2}=3$.

Table 3.1: Numerical results for different value of $p_{1}$ and $p_{2}$

\begin{tabular}{|cccccc|}
\hline$p_{1}$ & $p_{2}$ & $F_{1 ; i}>0$ & $\ldots$ & $F_{m_{k}-1 ; i}>0$ & $F_{m_{k} ; i} \leq 0$ \\
\hline 0.25001 & 0 & 2.4001 & $\ldots$ & $F_{246,1} \approx 8.607$ & $F_{247,1} \approx-26.30$ \\
0 & 0.14816 & 2.1109 & $\ldots$ & $F_{201 ; 2} \approx 25.93$ & $F_{202 ; 2} \approx-0.593$ \\
0.09018 & 0.09018 & 2.4373 & $\ldots$ & $F_{168 ; 2} \approx 22.76$ & $F_{169 ; 2} \approx-2.340$ \\
0.09 & 0.09047 & 2.4407 & $\ldots$ & $F_{47 ; 2} \approx 15.01$ & $F_{48 ; 2} \approx-4.5453$ \\
0.09047 & 0.09 & 2.4385 & $\ldots$ & $F_{172 ; 2} \approx 12.96$ & $F_{173 ; 2} \approx-6.733$ \\
\hline
\end{tabular}

For completeness, we give the algorithm on Matlab software used for making Table 3.1. It is available at https://octave-online.net. For three or more delay arguments, the running algorithm is is more complicated.

$$
n=200 ; p 1=0.09047 ; p 2=0.09 ; f 1=z \operatorname{eros}(1, n) ; f 2=z \operatorname{eros}(1, n)
$$




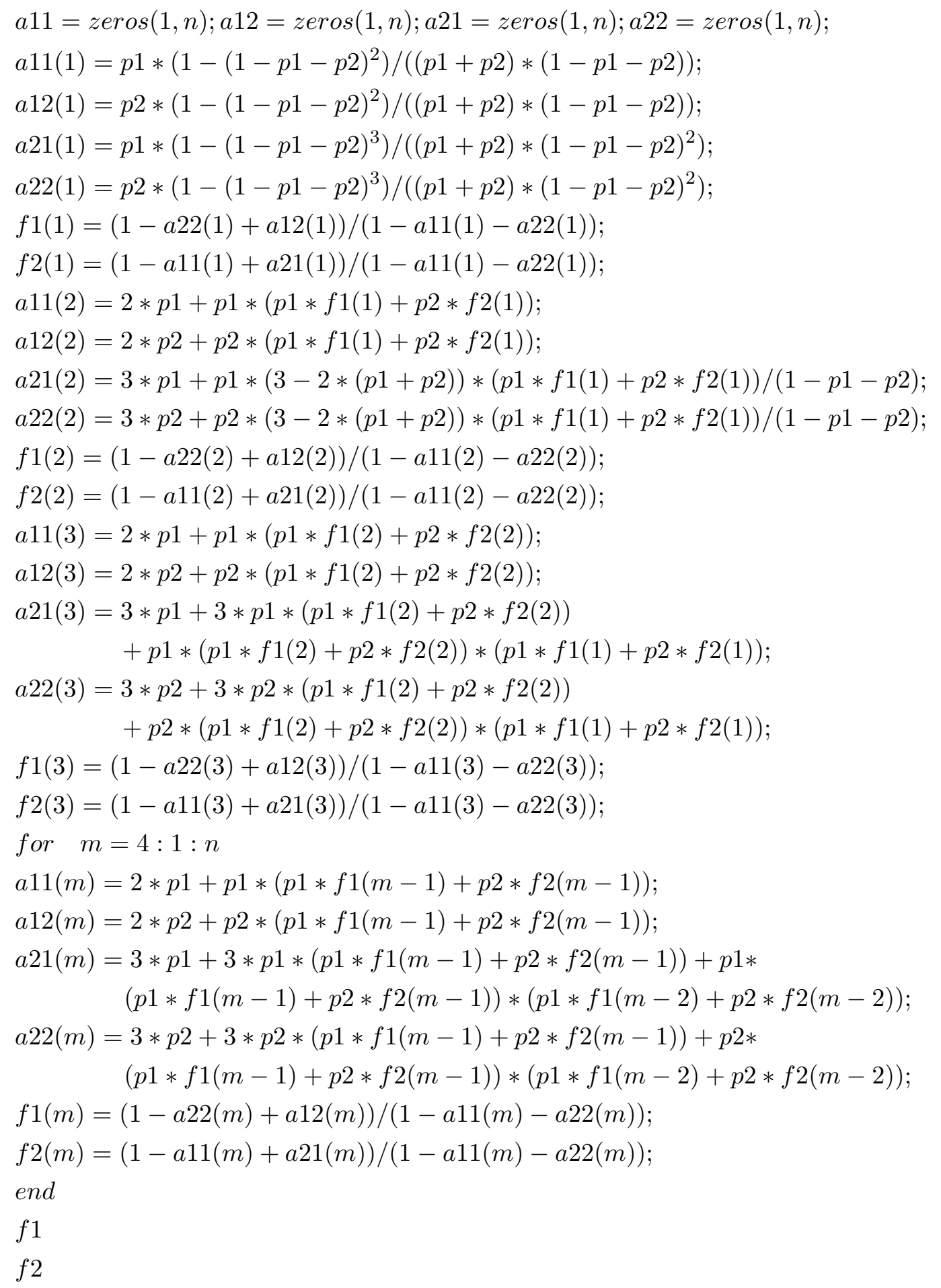




\section{Acknowledgments}

This project was supported by the Natural Science Foundation of Guangdong Province (No. 2018A0303130095) and the National Natural Science Foundation of China (NSFC: 71731006).

\section{References}

[1] Agarwal R. P., Bohner M., Grace S.R., O’Regan D.: Discrete Oscillation Theory, Hindawi Publishing Corporation. New York (2005).

[2] Braverman E., Johnson W.T.: On oscillation of difference equations with continuous time and variable delays. Appl. Math. Comput. 355, 449-457 (2019).

[3] Chatzarakis G.E., Pinelas S., Stavroulakis I.P.: Oscillations of difference equations with several deviated arguments, Aequat. Math. 88, 105-123 (2014).

[4] Chatzarakis G.E., Manojlovic J., Pinelas S., Stavroulakis I.P.: Oscillation criteria of difference equations with several deviated arguments, Yokohama Mathematical 60, 13-31 (2014).

[5] Chatzarakis G.E., Kusano T., Stavroulakis I.P.: Oscillation conditions for difference equations with several variable arguments, Math. Bohem. 140-3, 291-311 (2015).

[6] El-Morshed H.A.: On the distribution of zeros of solutions of first order delay difference equations, Nonlinear Anal. 74, 3353-3362 (2011).

[7] Goodrich C., Peterson A.: Discrete Fractional Calculus, Springer International Publishing, 2015.

[8] Ladas G., Stavroulakis I.P.: Oscillations caused by several retarded and advanced arguments, J. Difference Equations, 44, 134-152 (1982).

[9] Stavroulakis I.P.: Oscillations of delay difference equations, Comput. Math. Appl. 29, 83-88 (1995).

[10] Stavroulakis I.P.: Oscillation criteria for delay and difference equations with nonmonotone arguments, Appl. Math. Comput. 226, 661-672 (2014).

[11] Tang X.H., Yu J.S.: Oscillation of delay difference equations, Comput. Math. Appl. 37, 11-20 (1999).

[12] Tang X.H., Zhang R.Y.: New oscillation criteria for delay difference equations, Comput. Math. Appl. 42, 1319-1330 (2001). 
[13] Wu H.W., Chen C.R., Zhuang R. K.: Oscillation criterion for first-order delay differential equations with sign-changing coefficients, Electron. J. Differ. Equ. 126, $1-9$ (2017).

[14] Wu H.W., Dix J.G.: Oscillation criterion for first-order linear Differential equations with several delay arguments, Arab Journal of Mathematical Sciences 25-1, 43-56 (2019). 